$\underset{\text { clinical }}{\text { nephron }}$

Practice
Nephron 2019;143:184-187

DOI: 10.1159/000500168
Received: March 4, 2019

Accepted: April 4, 2019

Published online: May 3, 2019

\title{
Metabolic Stress Resistance in Acute Kidney Injury: Evidence for a PPAR-Gamma-Coactivator-1 Alpha-Nicotinamide Adenine Dinucleotide Pathway
}

\author{
Samir M. Parikh \\ Division of Nephrology and Center for Vascular Biology Research, Beth Israel Deaconess Medical Center and Harvard \\ Medical School, Boston, MA, USA
}

\section{Keywords}

Acute renal failure $\cdot$ Acute kidney injury $\cdot$ Mitochondria .

Tubule · Vitamin $B_{3} \cdot$ Nicotinamide $\cdot$ Niacinamide .

Nicotinamide adenine dinucleotide $\cdot$ Quinolinate

\begin{abstract}
Acute kidney injury (AKI) is estimated to affect $3-10 \%$ of all hospitalized adults in the United States, making it one of the most common inpatient diagnoses. Despite this staggering incidence, most individuals exposed to AKI stressors, such as intravenous radiocontrast or cardiopulmonary bypass, do not develop AKI. In fact, whereas animal models of ischemia, sepsis, or nephrotoxicity suggest near-uniform responses to stressors, the natural history of stressed patients is highly heterogeneous. Recent studies of mitochondrial perturbations underlying experimental and human AKI suggest a conserved metabolic contribution to this variance. The renal
\end{abstract}

tubule is only second to the heart in terms of mitochondrial abundance, reflecting the exquisite need for fuel combustion to generate the energy for active solute transport. The homeostasis of nicotinamide adenine dinucleotide (NAD+), a requisite coenzyme in oxidative metabolism, may be an important determinant of the renal response to AKI stressors. This mini-review highlights recent studies implicating NAD+ dysregulation in experimental and human AKI and summarizes findings from a pilot randomized trial to augment NAD+ among at-risk individuals.

(c) 2019 S. Karger AG, Basel

Contribution from the AKI and CRRT 2019 Symposium at the 24th International Conference on Advances in Critical Care Nephrology, Manchester Grand Hyatt, San Diego, CA, USA, February 26 - March 1, 2019. This symposium was supported in part by the NIDDK funded University of Alabama at Birmingham-University of California San Diego O'Brien Center for Acute Kidney Injury Research (P30DK079337).

\section{KARGER}

(c) 2019 S. Karger AG, Basel

E-Mail karger@karger.com

www.karger.com/nef
Samir M. Parikh

Beth Israel Deaconess Medical Center

330 Brookline Avenue, RN 330C

Boston, MA 02215 (USA)

E-Mail sparikh1@ bidmc.harvard.edu 


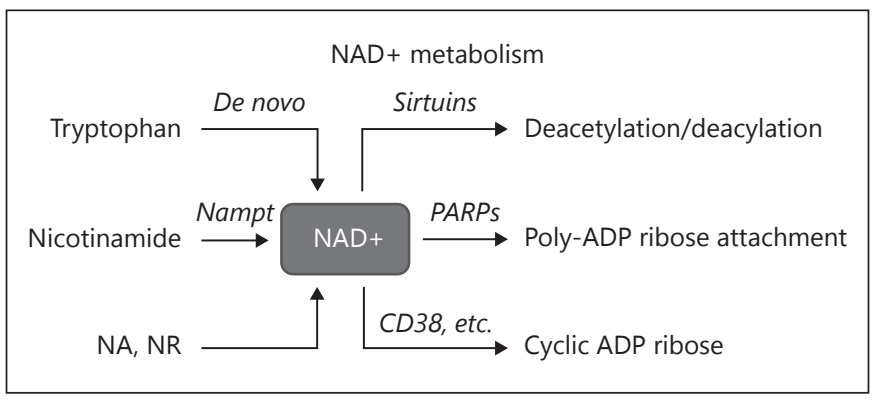

Fig. 1. Overview of NAD+ metabolism. NAD + is generated within cells from discrete dietary precursors: the essential amino acid tryptophan by a series of enzymes collectively referred to as the "de novo NAD+ biosynthesis pathway;" the vitamin B3 analog nicotinamide (also referred to as niacinamide) by the enzyme Nampt; and other precursors that include NA and NR. NAD+ is cleaved by enzymes of different classes: sirtuins, which remove acetyl or acyl modifications from proteins; PARPs, which add 1 or more poly-ADP ribose moieties to proteins; and ectonucleotidases such as CD38, which generate cyclic ADP ribose from $\mathrm{NAD}+$. NAD+, nicotinamide adenine dinucleotide; PARPs, polyADP ribose polymerases; NA, nicotinic acid; NR, nicotinamide riboside; Nampt, nicotinamide phosphoribosyltransferase.

Multiple strands of evidence implicate mitochondria at the nexus of renal health and disease. Classic tubular transport studies identify a linear relationship between filtration fraction and oxygen consumption; ultrastructural studies of human acute kidney injury (AKI) have consistently described early mitochondrial swelling in tubular epithelium; and single gene diseases affecting mitochondria exhibit a high prevalence of tubulopathy with progressive renal failure [1]. Thus, biochemical, pathological, and genetic studies all suggest that intact mitochondrial function is essential for normal tubular function, and deranged mitochondria are present in disease.

Along the nephron, the proximal tubule bears the largest burden of active solute transport. This segment preferentially combusts fatty acids to generate adenosine triphosphate. Fatty acid oxidation requires mitochondria and oxygen. Within mitochondria, the coenzyme nicotinamide adenine dinucleotide (NAD+) carries high-energy electrons from fatty acid oxidation to the electron transport chain. NAD+ is therefore a rate-limiting catalyst for fatty acid oxidation. This means that shortages in $\mathrm{NAD}+$ result in impaired energy extraction.

Intracellular NAD+ concentrations reflect a balance between biosynthesis from discrete dietary precursors and "consumption" by enzymes that cleave NAD+ to release nicotinamide (Fig. 1). Subcellular compartmentalization of NAD+ also appears to be important but is be- yond the current scope [2]. Stressors such as ischemia have been known to induce NAD+ consuming enzymes such as poly-ADP-ribose polymerases and CD 38 ; induction of these enzymes lowers NAD+ [3]. Tran and colleagues recently reported that renal tubular cell NAD+ levels are suppressed in models of AKI and that this reduction of NAD+ may blunt fatty acid oxidation, reduce adenosine triphosphate generation, and elevate susceptibility to AKI stressors [4].

Unexpectedly, they found that the stress-related fall in $\mathrm{NAD}+$ was not only a consequence of increased consumption but also the result of decreased biosynthesis. In seeking a mechanism of decreased NAD+ biosynthesis, this study identified a novel function of the mitochondrial biogenesis regulator PPAR-gamma-coactivator-1alpha (PGC1a) to coordinately induce the enzymes that sequentially convert the amino acid tryptophan to NAD+. This series of enzymes is collectively referred to as the "de novo" or "kynurenine" (one of the intermediates between tryptophan and NAD+) biosynthesis pathway for NAD+. This study showed that renal tubular PGC1a expression defends the nephron against unrelated stressors, and that artificial augmentation of NAD+ could effectively mimic PGC1a's salutary effects in the tubule. Hence, a common PGC1 $\alpha-N A D+$ "axis" was described for kidney protection across rodent models of ischemia, inflammation, and nephrotoxicity.

At least 2 biopsy-based studies proposed that human AKI syndromes also exhibit the suppression of PGC1 $\alpha[4$, 5], suggesting that the preclinical AKI findings could be amenable to translational investigation. Since biopsies are not practical for clinical AKI stratification and coactivator proteins such as PGC1 $\alpha$ are not attractive pharmaceutical targets, the link between PGC1 $\alpha$ and NAD+ biosynthesis has suggested new ways to assess energy metabolism and achieve PGC1 $\alpha$-like kidney protection.

Serendipitously, a metabolomics study of murine AKI urine specimens identified a signature highly specific for de novo NAD+ biosynthesis, namely, the elevation of urinary quinolinic acid (uQuin) [6]. Quin is a late intermediate in the de novo pathway and has no other metabolic fate other than to become NAD+ through the action of quinolinate phosphoribosyltransferase (QPRT) and subsequent enzymes. The elevation of uQuin suggested suppression of QPRT during AKI, consistent with the earlier study linking PGC1 $a$ to members of this pathway [4]. Reduced QPRT was then experimentally modeled by gene editing in mice. Strikingly, the urinary metabolome of QPRT haploinsufficient mice recapitulated nearly half of the urinary metabolic changes observed in experimental 
post-ischemic AKI. These mice were also highly susceptible to renal ischemia-reperfusion injury. Shortly thereafter, an independent study also identified de novo NAD+ biosynthesis as a pathway to fortify the kidney against ischemic and genotoxic stressors [7]. And a third study proposed that, in mammals, only the kidney and the liver exhibit appreciable de novo NAD+ biosynthesis [8].

This "reporter" function of uQuin in mice suggested a noninvasive way to monitor the PGC1a-NAD+ axismore specifically, de novo NAD+ biosynthesis-in people. A pilot case control study in cardiac surgery patients followed by a larger cohort study of critically ill patients confirmed that elevated uQuin preceded the clinical diagnosis of AKI [6]. Rising levels of uQuin were monotonically associated with increasing risk of AKI. Together, these results suggested that the perturbation of NAD+ biosynthesis observed in the controlled setting of murine ischemia-reperfusion injury was also present in the heterogeneous setting of clinical AKI.

The authors then embarked on a clinical trial to augment NAD+ among high-risk patients [6]. Since the de novo pathway appeared to be blocked by QPRT suppression, they chose to administer nicotinamide (also referred to as niacinamide), the base version of vitamin $\mathrm{B}_{3}$, which the team had shown to boost renal NAD+ through another biosynthetic route known as the "salvage" pathway [4]. The design was a Phase I-like pilot study that was randomized and placebo-controlled. The active arms received either 1 or $3 \mathrm{~g} /$ day of oral nicotinamide on the day prior to on-pump cardiac surgery, on the day of surgery, and on the first postoperative day. For comparison, the United States recommended a daily allowance of nicotinamide in adults is $\sim 15 \mathrm{mg} /$ day. The results showed that oral nicotinamide elevated circulating (and urinary) levels of nicotinamide. Because serum NAD+ may not reflect intracellular levels, the authors were unable to determine whether these doses boosted NAD+ itself. Nonetheless, they found no evidence of increased adverse events in the active arms and promising data that suggested nicotinamide-related protection from AKI. The trial was too small and the patient population was too well to detect differences in major renal endpoints, but the results pave the way for future clinical trials of therapeutic NAD+ augmentation.

PGC1 $\alpha$ has been studied in the context of tubular cell biology for nearly 15 years [9]. And the first renal genetic mouse model of PGC1 $\alpha$ isolating its benefit in AKI to the tubule was reported almost 9 years ago [10]. But the recent flurry of studies from multiple laboratories elucidating (a) the role of NAD+ downstream from PGC1a; (b) the contribution of de novo NAD+ biosynthesis to AKI resistance; and (c) the potential for treating AKI with $\mathrm{NAD}+$ augmentation should build great excitement for the many fundamental and clinical studies that are now needed $[4,6,7,11,12]$.

First, what is the set of critical downstream effectors from NAD+? A rate-limiting role in energy metabolism is clear, but could NAD+ also be modulating cell-regulatory enzymes such as sirtuins to achieve renal benefit? Second, are AKI risk factors such as aging and chronic kidney disease linked by imbalances in NAD+ biosynthesis versus consumption? Third, how robust is elevated uQuin as an indicator of waning ability to resist AKI stressors? In what setting(s) is this most important? More broadly, can urinary metabolic signatures enable practical risk stratification in future AKI trials? And finally, can pharmacological manipulations to boost the levels of NAD+ find clinical utility in the primary prevention or even treatment of AKI? If so, what are the optimal time windows and clinical contexts? Given that the heart and kidney share a reliance on mitochondrial fatty acid oxidation for normal function, it is also notable that cardiac injury also appeared to be diminished in the above trial, $\mathrm{NAD}+$ augmentation. These and many more questions merit investigation. The emerging data linking NAD+ balance to AKI resistance and the close conservation of these findings between experimental models and humans propose a new chapter in AKI research.

\section{Disclosure Statement}

S.M.P. is a consultant for Alkermes, Astellas, Cytokinetics, Mission Therapeutics, and Janssen; S.M.P. serves on the Scientific Advisory Board of Aerpio.

\section{Funding Sources}

Research Support: R01-DK095072, R35-HL139424, and R01AG1027002.

\footnotetext{
References 1 Emma F, Montini G, Parikh SM, Salviati L. Mitochondrial dysfunction in inherited renal disease and acute kidney injury. Nat Rev Nephrol. 2016 May;12(5):267-80.

2 Cambronne XA, Stewart ML, Kim D, JonesBrunette AM, Morgan RK, Farrens DL, et al. Biosensor reveals multiple sources for mitochondrialNAD ${ }^{+}$.Science.2016Jun;352(6292): 1474-7.
}

Parikh 
3 Ebrahimkhani MR, Daneshmand A, Mazumder A, Allocca M, Calvo JA, Abolhassani N, et al. Aag-initiated base excision repair promotes ischemia reperfusion injury in liver, brain, and kidney. Proc Natl Acad Sci USA. 2014 Nov;111(45):E4878-86.

4 Tran MT, Zsengeller ZK, Berg AH, Khankin EV, Bhasin MK, Kim W, et al. PGC1 $\alpha$ drives NAD biosynthesis linking oxidative metabolism to renal protection. Nature. 2016 Mar; 531(7595):528-32.

5 Drury ER, Zsengeller ZK, Stillman IE, Khankin EV, Pavlakis M, Parikh SM. Renal PGC1 $\alpha$ May Be Associated with Recovery after Delayed Graft Function. Nephron. 2018; 138(4):303-9.
6 Poyan Mehr A, Tran MT, Ralto KM, Leaf DE, Washco V, Messmer J, et al. De novo NAD+ biosynthetic impairment in acute kidney injury in humans. Nat Med. 2018 Sep;24(9): 1351-9.

7 Katsyuba E, Mottis A, Zietak M, De Franco F, van der Velpen V, Gariani K, et al. De novo $\mathrm{NAD}+$ synthesis enhances mitochondrial function and improves health. Nature. 2018 Nov;563(7731):354-9.

8 Liu L, Su X, Quinn WJ 3rd, Hui S, Krukenberg K, Frederick DW, et al. Quantitative Analysis of NAD Synthesis-Breakdown Fluxes. Cell Metab. 2018 May;27:1067-80.e5.

9 Rasbach KA, Schnellmann RG. Signaling of mitochondrial biogenesis following oxidant injury. J Biol Chem. 2007 Jan;282(4):235562.

10 Tran M, Tam D, Bardia A, Bhasin M, Rowe GC, Kher A, et al. PGC-1 $\alpha$ promotes recovery after acute kidney injury during systemic inflammation in mice. J Clin Invest. 2011 Oct; 121(10):4003-14

11 Guan Y, Wang SR, Huang XZ, Xie QH, Xu YY, Shang D, et al. Nicotinamide Mononucleotide, an NAD+ Precursor, Rescues Age-Associated Susceptibility to AKI in a Sirtuin 1-Dependent Manner. J Am Soc Nephrol. 2017 Aug;28(8):2337-52.

12 Kellum JA, Fuhrman DY. The handwriting is on the wall: there will soon be a drug for AKI. Nat Rev Nephrol. 2019 Feb;15(2):65-6. 